PHYSICAL REVIEW D 95, 049902(E) (2017)

\title{
Erratum: I-Love-Q relations for gravastars and the approach to the black-hole limit \\ [Phys. Rev. D 92, 124030 (2015)]
}

\author{
Paolo Pani \\ (Received 21 January 2017; published 9 February 2017)
}

\begin{abstract}
We point out two mistakes which affect the computation of the moment of inertia and of the tidal Love number in the original paper. Both mistakes have been corrected in Ref. [1] and the correct tidal Love number agrees with the recent computation of Ref. [2].
\end{abstract}

DOI: 10.1103/PhysRevD.95.049902

(1) Moment of inertia.-As noted in Ref. [1], the moment of inertia of a thin-shell gravastar with zero surface density is $I=M R^{2}$, and not $I=R^{3} / 2$ as stated in the original paper. Although these quantities agree in the black-hole limit, $R \rightarrow 2 M$, they generically differ from each other.

(2) Tidal Love numbers. - While Eqs. (25) and (26) are correct, the junction conditions (27) are wrong. As explained in Refs. [2,3], the correct junction conditions read

$$
[[K]]=0=\left[\left[d K / d r_{*}\right]\right],
$$

where the symbol "[[...]]" denotes the "jump" of a given quantity across the spherical shell, i.e. $[[A]] \equiv \lim _{\epsilon \rightarrow 0} A\left(r \rightarrow r_{0}+\epsilon\right)-A\left(r \rightarrow r_{0}-\epsilon\right)$. In the above expression, $K$ is a metric perturbation and $r_{*}$ is the tortoise coordinate; both quantities are defined in the original paper. By using the above junction condition, Eq. (32) in the original paper should be replaced by

$$
\begin{aligned}
\lambda(c)= & M^{5} \frac{16(1-2 c)^{2}}{\Gamma(c)}\left\{-2 c\left(-45+156 c+130 c^{2}-536 c^{3}+120 c^{4}\right)\right. \\
& +6 \sqrt{2 c}\left(-15+62 c-2 c^{2}-204 c^{3}+152 c^{4}+16 c^{5}\right) \tanh ^{-1}[\sqrt{2 c}] \\
& \left.-9(1-2 c)^{3}\left(-5-6 c+6 c^{2}+4 c^{3}\right) \tanh ^{-1}[\sqrt{2 c}]^{2}\right\},
\end{aligned}
$$

with $c=M / R$ and

$$
\begin{aligned}
\Gamma(c)= & 15(1-2 c)^{2}\left\{2 c(-3+10 c)\left(2 c\left(-45-39 c+96 c^{2}+32 c^{3}+32 c^{4}\right)+\left(-45+6 c+150 c^{2}-36 c^{3}\right) \log [1-2 c]\right)\right. \\
& +9(1-2 c)^{2} \tanh ^{-1}[\sqrt{2 c}]^{2}\left(2 c\left(15+3 c-46 c^{2}-16 c^{3}\right)+3\left(5-4 c-18 c^{2}+8 c^{3}+8 c^{4}\right) \log [1-2 c]\right) \\
& +6 \sqrt{2 c} \tanh ^{-1}[\sqrt{2 c}]\left(2 c\left(-45+141 c+120 c^{2}-460 c^{3}+64 c^{4}+64 c^{6}\right)\right. \\
& \left.\left.+3\left(-15+62 c-2 c^{2}-204 c^{3}+152 c^{4}+16 c^{5}\right) \log [1-2 c]\right)\right\}
\end{aligned}
$$

The above result agrees with that derived in Ref. [1] using a different approach and also with the result of Ref. [2].

The most striking difference between Eq. (2) and the wrong one in the original paper is evident in the black-hole limit. While the tidal Love number derived in the original paper vanishes as $\lambda \sim(1 / 2-c)^{2}$ as $c \rightarrow 1 / 2$ [cf. Eq. (34)] the correct result in Eq. (2) vanishes logarithmically, as discussed in Ref. [2].

N. Uchikata, S. Yoshida, and P. Pani, Phys. Rev. D 94, 064015 (2016).

V. Cardoso, E. Franzin, A. Maselli, P. Pani, and G. Raposo, arXiv:1701.01116.

P. Pani, E. Berti, V. Cardoso, Y. Chen, and R. Norte, Phys. Rev. D 80, 124047 (2009). 